ARTICLE OPEN

Check for updates

\title{
Ductal carcinoma in situ: a risk prediction model for the underestimation of invasive breast cancer
}

\author{
Ko Woon Park (iD), Seon Woo Kim ${ }^{2}$, Heewon Han ${ }^{2}$, Minsu Park $\mathbb{D}^{3}$, Boo-Kyung Han ${ }^{1}$, Eun Young Ko ${ }^{1}$, Ji Soo Choi ${ }^{1}$, Eun Yoon Cho ${ }^{4}$, \\ Soo Youn Cho iD ${ }^{4}$ and Eun Sook Ko (iD ${ }^{1 凶}$
}

Patients with a biopsy diagnosis of ductal carcinoma in situ (DCIS) may be diagnosed with invasive breast cancer after excision. We evaluated the preoperative clinical and imaging predictors of DCIS that were associated with an upgrade to invasive carcinoma on final pathology and also compared the diagnostic performance of various statistical models. We reviewed the medical records; including mammography, ultrasound (US), and magnetic resonance imaging (MRI) findings; of 644 patients who were preoperatively diagnosed with DCIS and who underwent surgery between January 2012 and September 2018. Logistic regression and three machine learning methods were applied to predict DCIS underestimation. Among 644 DCIS biopsies, 161 (25\%) underestimated invasive breast cancers. In multivariable analysis, suspicious axillary lymph nodes (LNs) on US (odds ratio [OR], 12.16; 95\% confidence interval $[\mathrm{Cl}], 4.94-29.95 ; P<0.001)$ and high nuclear grade $(\mathrm{OR}, 1.90 ; 95 \% \mathrm{Cl}, 1.24-2.91 ; P=0.003)$ were associated with underestimation. Cases with biopsy performed using vacuum-assisted biopsy (VAB) $(\mathrm{OR}, 0.42 ; 95 \% \mathrm{Cl}, 0.27-0.65 ; P<$ $0.001)$ and lesion size $<2 \mathrm{~cm}$ on mammography $(\mathrm{OR}, 0.45 ; 95 \% \mathrm{Cl}, 0.22-0.90 ; P=0.021)$ and $\mathrm{MRI}(\mathrm{OR}, 0.29 ; 95 \% \mathrm{Cl}, 0.09-0.94 ; P=$ 0.037 ) were less likely to be upgraded. No significant differences in performance were observed between logistic regression and machine learning models. Our results suggest that biopsy device, high nuclear grade, presence of suspicious axillary LN on US, and lesion size on mammography or MRI were independent predictors of DCIS underestimation.

npj Breast Cancer (2022)8:8; https://doi.org/10.1038/s41523-021-00364-z

\section{INTRODUCTION}

Ductal carcinoma in situ (DCIS) is a preinvasive or noninvasive breast cancer defined as the proliferation of neoplastic cells within the mammary ducts without invasion into the surrounding tissue ${ }^{1}$. DCIS accounts for almost $30 \%$ of newly diagnosed breast cancers ${ }^{2}$. DCIS underestimation is defined as the failure to detect invasive cancer in a preoperative biopsy, with the actual diagnosis becoming evident only after a pathological examination of the surgical specimen ${ }^{3}$. The reported risk of underestimation varies from 14 to $43 \% 4,5$, with one meta-analysis estimating the risk at $25.9 \%{ }^{6}$. The standard treatment for patients diagnosed with DCIS is wide local excision with radiation or mastectomy ${ }^{7,8}$. However, due to concerns regarding DCIS underestimation, routine sentinel lymph node biopsy (SLNB) may be necessary in patients with DCIS diagnosed by core needle biopsy $(\mathrm{CNB})^{9,10}$. Unfortunately, axillary dissection is often accompanied by complications such as pain, numbness, and arm swelling ${ }^{11}$. Therefore, the preoperative prediction of upgraded diagnosis to invasive cancer could avoid unnecessary axillary surgery, including SLNB.

Studies have attempted to identify the risk factors for underestimation, including nuclear grade and radiological findings such as lesion size on imaging, mass on mammography or ultrasound (US), and final Breast Imaging Reporting and Data System (BIRADS) assessment categories ${ }^{2,4-6,10,12-15}$. Previous studies have also revealed an association between the risk of underestimation and factors such as age, palpability, histologic suspicion of invasion, imaging guidance method, biopsy device, and other factors. Although several papers have analyzed or mentioned all three imaging findings (mammography, ultrasound [US], and magnetic resonance imaging $[\mathrm{MRI}]$ ), they didn't describe a prediction model or just evaluated limited imaging findings ${ }^{15-17}$. To our knowledge, there is no study for evaluating prediction model using all imaging findings (mammography, US, and MRI)

Machine learning (ML) is a computational method capable of learning to improve the performance of a task based on previous experience. The ML field is closely related to pattern recognition and statistical inference and has been applied to problems across many fields, including bioinformatics ${ }^{18}$. ML overcomes or reduces the impact of the limitations of commonly used statistical techniques, which usually consider a limited finite set of hypotheses in their evaluations. However, ML approaches generate models for prediction by extensively searching the model and parameter space; thus, these approaches have been adopted for predictive modeling and decision-making in biomedicine ${ }^{19}$. However, there are few reports on the use of ML techniques for the prediction of DCIS underestimation or examining the potential improvement in prediction performance using $\mathrm{ML}^{11}$.

Therefore, the present study aimed to identify clinicopathologic and imaging features that predicted an upgrade of DCIS to invasive carcinoma on final pathologic diagnosis and to compare the diagnostic performance of various statistical models, including $\mathrm{ML}$ techniques.

\section{RESULTS}

\section{Factors associated with DCIS underestimation}

Among 688 patients with biopsy-confirmed DCIS in our institution, we included 644 patients (mean age, 51.4; range, 22-87 years)

${ }^{1}$ Department of Radiology, Samsung Medical Center, Sungkyunkwan University School of Medicine, Seoul, Republic of Korea. ${ }^{2}$ Statistics and Data Center, Research Institute for

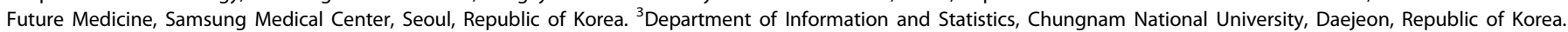
${ }^{4}$ Department of Pathology and Translational Genomics, Samsung Medical Center, Sungkyunkwan University School of Medicine, Seoul, Republic of Korea.

email: mathilda0330@gmail.com 


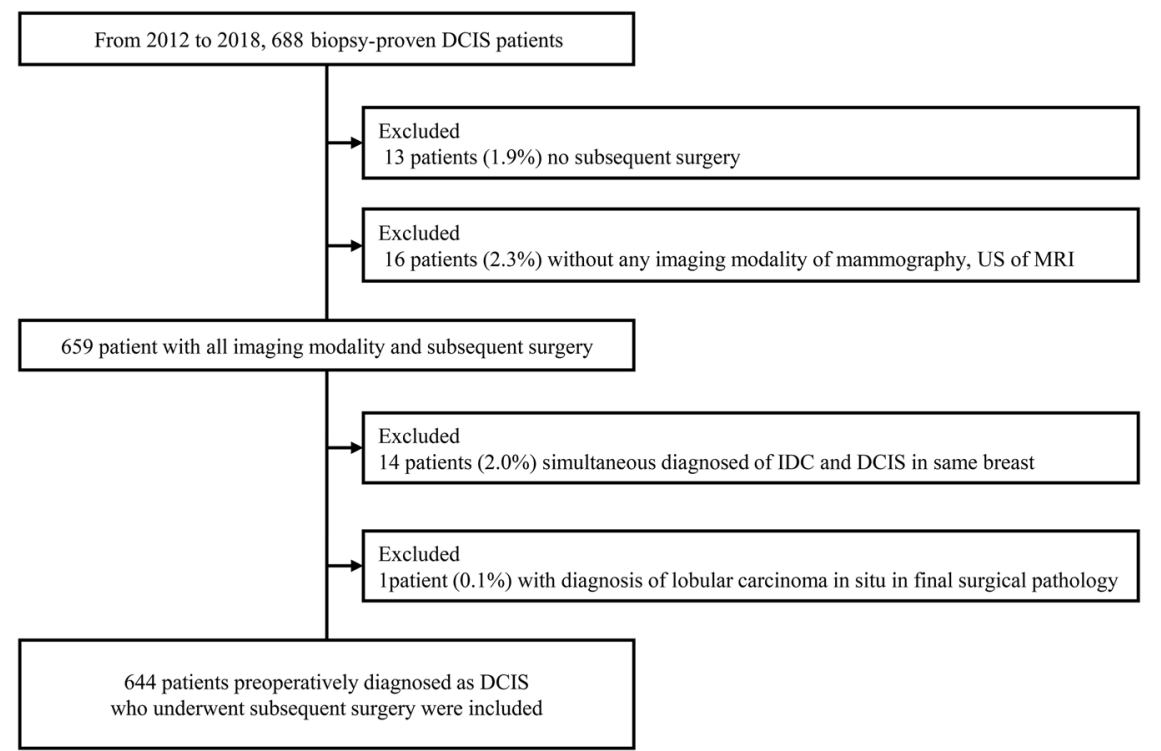

Fig. 1 Flow chart of the study population. After we reviewed the biopsy database for biopsy-confirmed DCIS at our institution, we identified 688 biopsy-proven DCIS. This flowchart briefly presents how many patients were excldued and the reasons of exclusion.

who underwent subsequent surgery. Forty-four patients were excluded for the reasons shown in Fig. 1. Of the 644 DCIS lesions subjected to biopsy, 161 (25\%) were underestimated invasive ductal carcinoma (IDC), including 73 identified as microinvasive cancers after surgery. The mean size of invasive cancer from surgical specimens was $3.89 \mathrm{~mm}$ (range, 0.01-35 mm, $\pm 6.1 \mathrm{~mm}$ ). Tables 1 and 2 show the patient characteristics and univariable analysis of the factors associated with the underestimation of invasive carcinoma. Palpability $(P<0.001)$ and lesion size $>2 \mathrm{~cm}$ $(P<0.001)$ were significantly associated with histologic upgrade. Cases with mammography guidance $(P<0.001)$, vacuum-assisted biopsy (VAB) device $(P<0.001)$, thicker biopsy needle $(P<0.001)$, and a larger number of specimens $(P=0.001)$ were less likely to be underestimated. Pathologically, high nuclear grade $(P<0.001)$ and the presence of comedo necrosis $(P<0.001)$ were associated with underestimation. Imaging findings with cancer not visible on US or MRI resulted in significantly lower underestimation $(P<$ 0.001 and $P<0.001$, respectively). Mammographic findings indicated that combined mass/focal asymmetry with microcalcifications occurred significantly more frequently in the IDC group $(P=0.002)$. Fine linear/fine branching microcalcifications $(P<$ $0.001)$ and linear/segmental distribution $(P<0.001)$ were also observed significantly more frequently in the IDC group. US findings showed that the presence of microcalcifications $(P<$ $0.029)$, irregular mass shape $(P<0.001)$, suspicious axillary LN $(P<0.001)$, and high vascularity $(P=0.001)$ were significant indicators of underestimation MRI findings of irregular mass shape $(P=0.027)$, linear/segmental distribution of non-mass enhancement (NME) $(P=0.022)$, clustered ring enhancement pattern of NME $(P=0.003)$, and the presence of a washout pattern in the delayed phase of the time-intensity curve $(P=$ 0.002) were significantly associated with underestimation.

The results of the multivariable analysis showed a prediction model with the highest Nagelkerke $R^{2}$ index (0.205, range; 0.161-0.205) and the smallest Akaike's Information Criteria (AIC) (592.221, range; 592.221-627.243) including a biopsy device, nuclear grade, the presence of suspicious LNs on US, and lesion size on mammography and MRI. Underestimation was associated with suspicious axillary LN on US (odds ratio [OR], 12.16; 95\% confidence interval $[\mathrm{Cl}], 4.94-29.95 ; P<0.001)$ and high nuclear grade (OR, 1.90; 95\% Cl, 1.24-2.91; $P=0.003)$. Biopsy performed using $V A B(O R, 0.42 ; 95 \% \mathrm{Cl}, 0.27-0.65 ; P<0.001)$, lesion size
$<2 \mathrm{~cm}$ (OR, 0.45; 95\% Cl, 0.22-0.90; $P=0.021)$, and non-visible on mammography (OR, $0.41 ; 95 \% \mathrm{Cl}, 0.22-0.76, P=0.002)$ and lesion size $<2 \mathrm{~cm}(\mathrm{OR}, 0.29 ; 95 \% \mathrm{Cl}, 0.09-0.94 ; P=0.037)$ or non-visible on MRI (OR, $0.52 ; 95 \% \mathrm{Cl}, 0.28-0.95 ; P=0.031)$ were less likely to be underestimated (Table 3 and Supplementary Figs. 1, 2). Six different models that included up to six risk factors were used for adjustment according to various combinations. The combinations of risk factors for each model and their values are shown in the Supplementary Table (online). Among them, Model 6 showed the best performance (Fig. 2).

\section{Performances of the prediction models}

The four prediction models based on logistic regression and three ML techniques showed similar diagnostic performance (Table 4). All four methods showed similar results, with no significant differences in predicting DCIS underestimation. The area under the curves (AUCs) of the four models ranged from 0.66 to 0.78 . The three ML methods predicted the risk factors in descending order shown in Fig. 3. All four models reported that the most important risk factor was suspicious axillary LN on US, followed by lesion size on MRI, (except for the random forest technique). In the decision tree technique, only suspicious axillary LNs on US, lesion size on MRI, and biopsy device were significant risk factors, whereas lesion size on mammography and nuclear grade were not used for classification.

\section{DISCUSSION}

The rate of underestimation of DCIS by percutaneous biopsy varies from $14 \%$ to $43 \%$ depending on the imaging guidance and needle gauge ${ }^{4-6}$. The overall DCIS underestimation rate of $25 \%$ $(161 / 644)$ in our study was within this reported range. Of the 161 invasive carcinomas, 73 were revealed as microinvasive carcinoma $(11.3 \%, 73 / 644)$. It was a relatively higher incidence than the previously reported number, $\sim 5-10 \%$ of cases of $\mathrm{DCIS}^{20}$. The higher proportion of cases with microinvasion was likely because the pathology slides are thoroughly read through intensive sampling. The lower proportion of frank invasive cancer compared to that in previous studies may have been because the biopsies were performed by experienced breast radiologists (more than 5 years of experience) in most cases. 


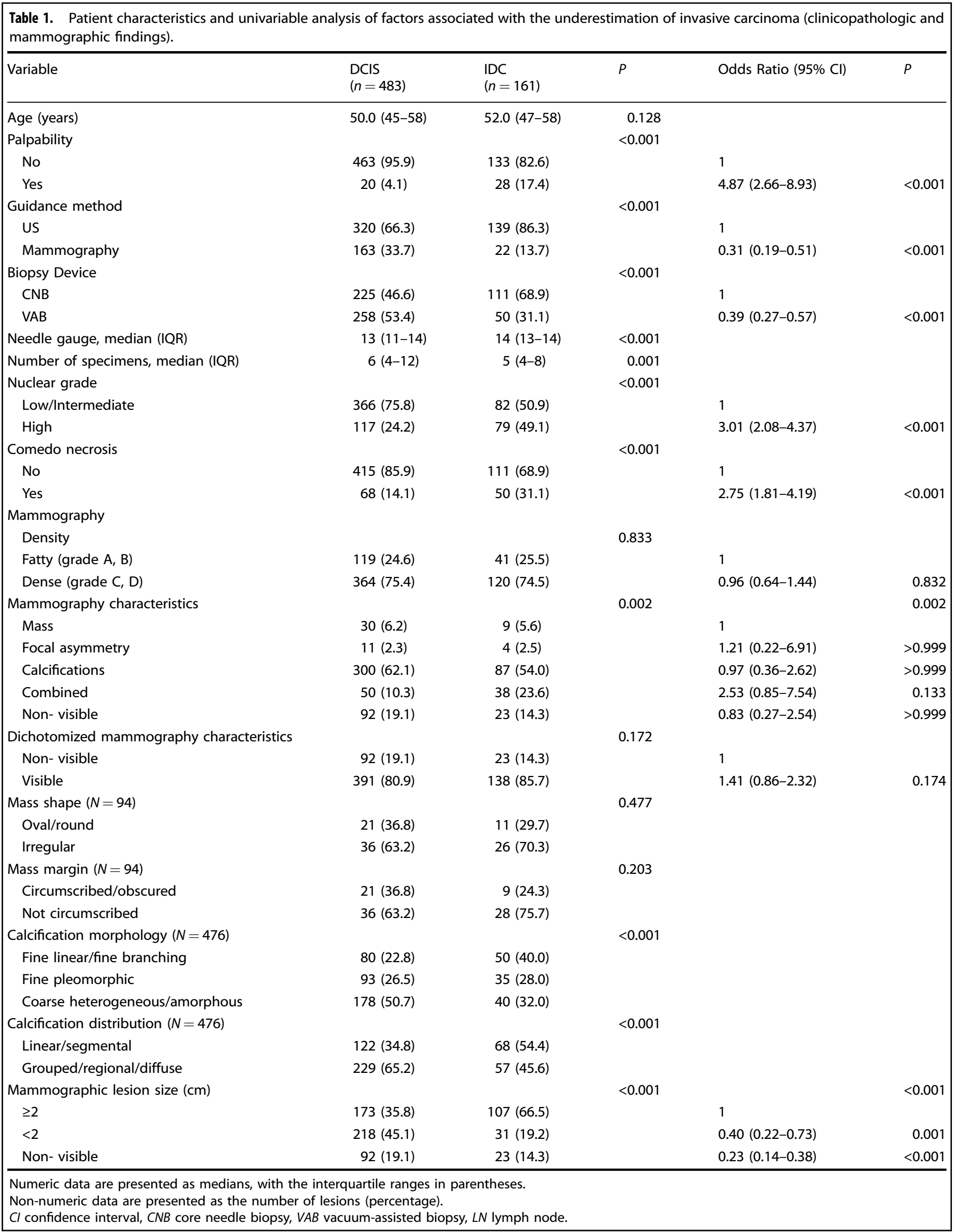


Table 2. Patient characteristics and univariable analysis of factors associated with the histopathologic upgrade to invasive carcinoma (US and MRI findings).

\begin{tabular}{|c|c|c|c|c|c|}
\hline Variable & $\operatorname{DCIS}(n=483)$ & $\operatorname{IDC}(n=161)$ & $P$ & Odds Ratio $(95 \% \mathrm{Cl})$ & $P$ \\
\hline US characteristics & & & $<0.001$ & & $<0.001$ \\
\hline Non-mass & $178(36.8)$ & $86(53.4)$ & & $0.33(0.17-0.64)$ & $<0.001$ \\
\hline Non-visible & $149(30.9)$ & $18(11.2)$ & & $1.32(0.84-2.08)$ & 0.337 \\
\hline Visible & $334(69.1)$ & $143(88.8)$ & & $3.54(2.09-6.00)$ & $<0.001$ \\
\hline Calcifications on US $(N=477)$ & & & 0.029 & & \\
\hline No & $148(44.3)$ & 48 (33.6) & & & \\
\hline Yes & $186(55.7)$ & $95(66.4)$ & & & \\
\hline Mass shape $(N=213)$ & & & $<0.001$ & & \\
\hline Not circumscribed & $116(74.4)$ & $48(84.2)$ & & & \\
\hline Mass orientation $(N=213)$ & & & 0.821 & & \\
\hline Parallel & $135(86.5)$ & $50(87.7)$ & & & \\
\hline Nonparallel & $21(13.5)$ & 7 (12.3) & & & \\
\hline Echo pattern $(N=477)$ & & & 0.136 & & \\
\hline Isoechoic & $91(27.3)$ & $26(18.2)$ & & & \\
\hline Hypoechoic & $232(69.5)$ & $113(79.0)$ & & & \\
\hline Hyperechoic & $1(0.3)$ & $0(0)$ & & & \\
\hline Complex echoic & $10(2.9)$ & $4(2.8)$ & & & \\
\hline Posterior acoustic features $(N=477)$ & & & 0.338 & & \\
\hline Not available & $25(7.5)$ & $16(11.2)$ & & & \\
\hline Suspicious axillary LN & & & $<0.001$ & & \\
\hline No & $476(98.6)$ & $127(78.9)$ & & 1 & \\
\hline Yes & $7(1.4)$ & $34(21.1)$ & & $18.21(7.89-42.03)$ & $<0.001$ \\
\hline US lesion size $(\mathrm{cm})$ & & & $<0.001$ & & $<0.001$ \\
\hline$\geq 2$ & $113(23.4)$ & $87(54.0)$ & & 1 & \\
\hline$<2$ & $221(45.8)$. & $56(34.8)$ & & $0.16(0.08-0.30)$ & $<0.001$ \\
\hline Non- visible & $149(30.8)$ & $18(11.2)$ & & $0.33(0.21-0.52)$ & $<0.001$ \\
\hline \multicolumn{6}{|l|}{ MRI } \\
\hline MRI characteristics & & & $<0.001$ & & 0.001 \\
\hline Mass & $142(29.4)$ & $52(32.3)$ & & 1 & \\
\hline Non-mass enhancement & $262(54.2)$ & $104(64.6)$ & & $0.17(0.06-0.52)$ & $<0.001$ \\
\hline Non-visible & $79(16.4)$ & $5(3.1)$ & & $1.08(0.69-1.69)$ & $>0.999$ \\
\hline Dichotomized MRI characteristics & & & $<0.001$ & & \\
\hline Non-visible & $79(16.4)$ & $5(3.1)$ & & 1 & \\
\hline Visible & 404 (83.6) & $156(96.9)$ & & $6.10(2.43-15.35)$ & $<0.001$ \\
\hline Mass shape $(N=194)$ & & & 0.027 & & \\
\hline Oval/round & 34 (23.9) & $5(9.6)$ & & & \\
\hline Irregular & $108(76.1)$ & $47(90.4)$ & & & \\
\hline
\end{tabular}




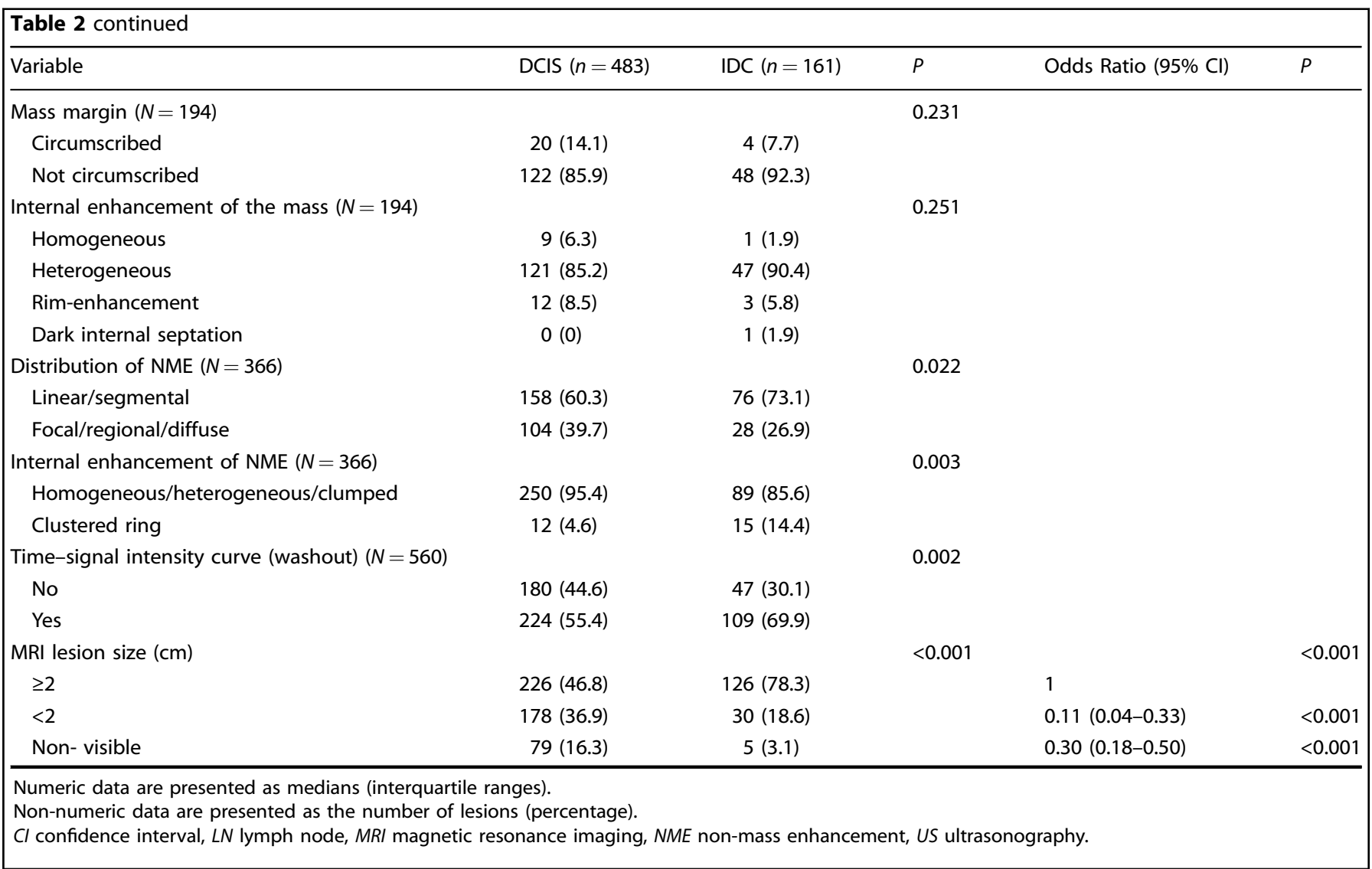

\begin{tabular}{|c|c|c|}
\hline Variable & Odds ratio $(95 \% \mathrm{Cl})$ & $P$ \\
\hline \multicolumn{3}{|l|}{ Device } \\
\hline CNB & 1 & \\
\hline VAB & $0.42(0.27-0.65)$ & $<0.001$ \\
\hline \multicolumn{3}{|l|}{ Nuclear grade } \\
\hline Low/Intermediate & 1 & \\
\hline High & $1.90(1.24-2.91)$ & 0.003 \\
\hline \multicolumn{3}{|l|}{ Suspicious LN on US } \\
\hline No & 1 & \\
\hline Yes & $12.16(4.94-29.95)$ & $<0.001$ \\
\hline Lesion size on mammography $(\mathrm{cm})$ & & 0.002 \\
\hline$\geq 2$ & 1 & \\
\hline$<2$ & $0.45(0.22-0.90)$ & 0.021 \\
\hline Non-visible & $0.41(0.22-0.76)$ & 0.002 \\
\hline Lesion size on MRI (cm) & & 0.008 \\
\hline$\geq 2$ & 1 & \\
\hline$<2$ & $0.29(0.09-0.94)$ & 0.037 \\
\hline Non- visible & $0.52(0.28-0.95)$ & 0.031 \\
\hline
\end{tabular}

Compared to previous studies on DCIS underestimation, our study included all three imaging modalities (e.g., mammography, US, and MRI) and a large number of cases of biopsy-confirmed DCIS ( $n=644), 161$ of which were upgraded to invasive cancer.

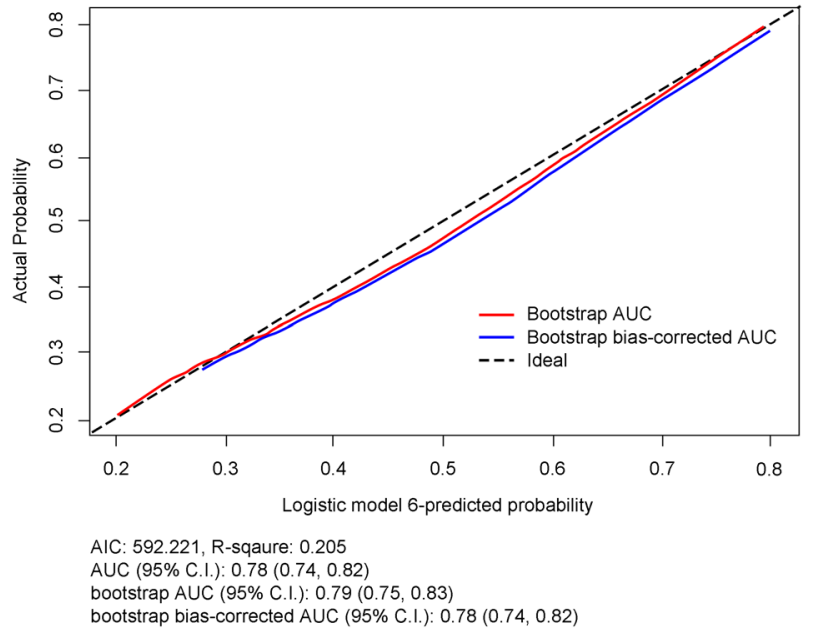

Fig. 2 Calibration curve to predict the histologic upgrade of logistic model 6 . Notes: The $\mathrm{x}$-axis represents the predicted upgrade risk. The $y$-axis represents the actual histologic upgrade. The diagonal dotted line represents a perfect prediction by an ideal model. The solid line represents the performance of model 6 . The closer the solid line is to the diagonal, the more accurate the prediction.

These features make this the largest study to describe the relationship between imaging findings and underestimation, with more meaningful results obtained through a large number of patients and data from all three imaging modalities. Our results revealed a significantly lower upgrade for mammography than that for US guidance $(P<0.001)$. This is because mammographic guidance is almost always used for lesions that present as 
calcifications only (without mass), which is associated with the absence of invasion compared to invasive cancer ${ }^{18}$ and, thus, would be consistent with an ultimate diagnosis of DCIS only. Similar to previous studies ${ }^{4,5,21}$, our results revealed that biopsy with VAB $(P<0.001)$, larger sample numbers $(P=0.001)$, and thicker biopsy needles $(P<0.001)$ were associated with a lower occurrence of histopathologic upgrade. Notably, non-visible lesions on US or MRI were less likely to be upgraded to invasive cancer $(P<0.001)$, whereas non-visible lesions on mammography were not significantly associated with the upgrade $(P=0.172)$.

Table 4. Comparisons of AUCs between four prediction models.

\begin{tabular}{lll}
\hline & AUC & $95 \% \mathrm{Cl}$ \\
\hline Logistic regression & 0.78 & $0.74-0.82$ \\
Decision tree & 0.75 & $0.56-0.93$ \\
Bagging & 0.66 & $0.50-0.83$ \\
Random forest & 0.75 & $0.58-0.91$ \\
\hline
\end{tabular}

$A U C$ area under the receiver operating characteristic curve, $\mathrm{Cl}$ confidence interval.
We speculate that many lesions could be masked on mammography because most Asian patients have dense breasts.

Our results revealed a relationship between DCIS with invasive components and the presence of suspicious axillary LNs on US $(P<0.001)$. Similar to the previous reports ${ }^{10,14,22}$, clustered ring enhancement of NME $(P=0.003)$ and a washout kinetic pattern at the delayed phase $(P=0.002)$ on MRI were frequently observed in the IDC group. Our results also confirmed that a larger lesion size on mammography or MRI was a predictive factor for the underestimation of invasive cancer ${ }^{6}$. A larger lesion size on mammography was previously reported as an independent predictive factor for invasion, with a cutoff ranging from 20 to $60 \mathrm{~mm}^{2,23}$. These features could reflect the assumption that DCIS and invasive cancer are more likely to coexist in large lesions. Moreover, the results of the multivariable analysis revealed high nuclear as a risk factor for underestimation, consistent with previous reports ${ }^{14,15}$

We applied three ML methods and logistic regression analysis to assess the underestimation risk. ML techniques did not significantly improve the prediction of underestimation. Although the mean size of an upgraded invasive cancer was $3.89 \mathrm{~mm}$ in our study, relatively smaller than those reportedly previously ${ }^{12,15}$, the AUCs were relatively good for all models (0.66-0.78).

\section{a Decision tree}

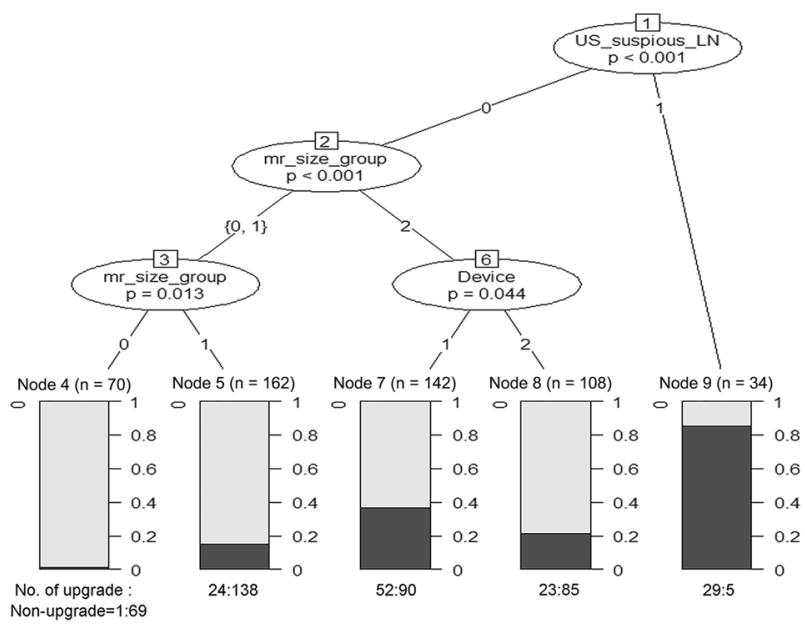

c Random forest

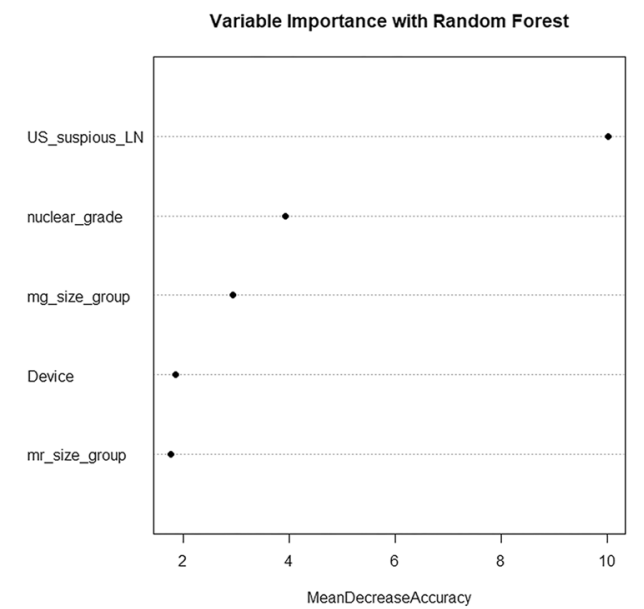

b Bagging

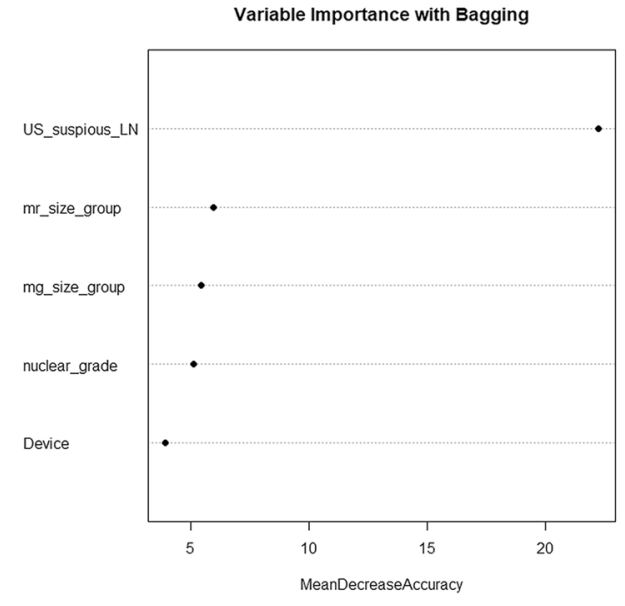

d Nomogram for logistic regression

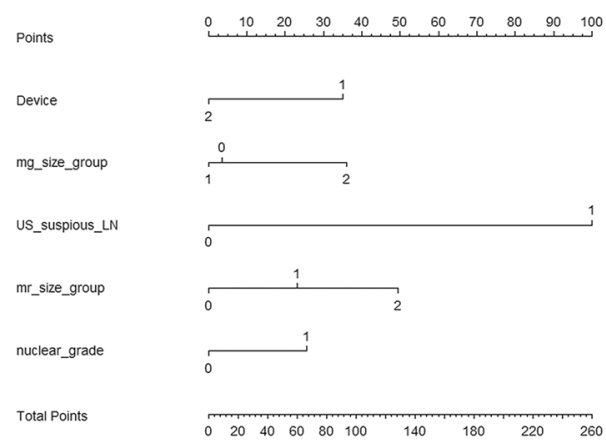

Predicted Value

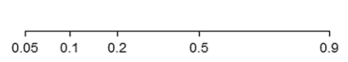

Fig. 3 Variable importance graphs of the most important risk factors in descending order and nomogram. a decision tree, $\mathbf{b}$ bagging, and c random forest-based machine learning methods and $\mathbf{d}$ a nomogram for the logistic regression model. 
Our study has some limitations. First, we did not perform an observer study involving multiple readers. Second, most of our patients routinely underwent all three imaging studies in their preoperative evaluations. However, this could differ according to national guidelines or insurance coverage in other countries. Therefore, our results may not be generalizable or reflect all clinical conditions.

In conclusion, the biopsy device, high nuclear grade, the presence of suspicious axillary LN on US, and lesion size $>2 \mathrm{~cm}$ on mammography or MRI were independent predictors of DCIS underestimation. We observed no significant differences in performance between the conventional prediction and $\mathrm{ML}$ models.

\section{METHODS \\ Patients}

The Institutional Review Board of Samsung Medical Center approved this retrospective study (SMC IRB 2019-12-077-001) and waived the requirement for informed consent due to the retrospective nature of this study. Between January 2012 and September 2018, we reviewed the biopsy database for biopsy-confirmed DCIS at our institution. Once DCIS is diagnosed using biopsy, the standard practice at our institution is to perform mammography, US, and MRI as preoperative imaging workup in all patients. We excluded patients who had not undergone subsequent surgery; without any imaging modality among mammography, US, or MRI; and who had been simultaneously diagnosed with IDC and DCIS in the same breast.

\section{Biopsy procedure}

All needle biopsies were performed using imaging guidance by one of the eight radiologists with 1-26 years of breast imaging experience. US-guided CNB was performed using a 14-or 18-gauge (G) Tru-cut needle with a $22 \mathrm{~mm}$ throw (ACECUT, TSK Laboratory, Tokyo, Japan), with a minimum of four cores obtained from each lesion. VABs were performed for small or non-mass lesions or lesions containing calcifications. VABs were also indicated when precise targeting was difficult by the core needle or the in cases in which the results might vary depending on the amount of tissue sample. US-guided VAB was performed using an 8-18-G vacuum-assisted probe (Mammotome, Devicor Endo-Surgery, Cincinnati, $\mathrm{OH}$; Suros, Hologic Inc. Bedford, MA). The needle gauge was determined by lesion size or characteristics and each radiologist's preference. Stereotactic VAB was performed for microcalcifications that were not visible on US, using an 11-G vacuum-assisted probe (Mammotome, Devicor Endo-Surgery, Cincinnati, $\mathrm{OH}$ ) and the stereotactic unit of a prone table (Lorad, Hologic Inc., Danbury, (T).

\section{Data and image analysis}

Radiologic variables were collected by reviewing each image retrospectively in consensus by two radiologists (initials blinded) with nine and 14 years of experience in breast imaging who were blinded to the final pathologic outcome. The BI-RADS lexicon was used to describe the mammographic, US, and MRI features ${ }^{24}$. In cases in which the lesion showed no imaging findings, the lesion characteristics were classified as non-visible. The lesion sizes were dichotomized to evaluate the effect of size on the upgrade to invasive cancer by setting a cutoff value of $2 \mathrm{~cm}$, as reported previously $y^{5,6,25}$. The following mammography features were evaluated: breast density, lesion characteristics (mass/focal asymmetry, calcifications, combined or non-visible), and lesion size. A mass detected on mammography was evaluated for its shape (oval/round or irregular) and margins (circumscribed/obscured or not circumscribed). Calcifications on mammography were assessed for their morphology (fine linear/ branching, fine pleomorphic, coarse heterogeneous/amorphous, or benign appearance) and distribution (linear/segmental or grouped/ regional/diffuse).

The following US features were also evaluated: lesion characteristics (mass, non-mass lesion [NML], or non-visible), lesion size, shape (oval/ round or irregular), margin (circumscribed or not circumscribed), orientation (parallel or nonparallel), echo pattern (isoechoic, hypoechoic, hyperechoic, or complex/heterogeneous echoic), posterior acoustic features (no posterior feature, enhancement, shadowing, or combined), presence of suspicious axillary LNs, calcifications, and vascularity. An NML was defined as a focal hypoechoic area presenting as a confined asymmetry on two orthogonal planes that could not be characterized as a distinct mass owing to the lack of conspicuous margins or shape that also differed from the surrounding glandular tissue ${ }^{26}$. In addition, the vascularity on color Doppler US was determined according to the number of vessels within or around the lesion and was categorized as low (no flow or only one vessel flow signal observed) or high (more than two vessel flow signals observed $)^{27}$.

Dynamic contrast-enhanced MRI (DCE-MRI) data were reviewed for lesion size, lesion characteristics (mass, NME, or non-visible), lesion morphology (shape, margin, and internal enhancement in mass; distribution and internal enhancement in NME), and time-signal intensity curve pattern. Time-signal intensity curve patterns were categorized based on the presence of washout in the delayed phase. The imaging characteristics in all three modalities were also dichotomized as visible or non-visible to assess whether visibility affected upgrade. The interval between the initial diagnosis and operation was within 1 month in all patients.

All pathologic specimens including biopsy and surgery were read by two experienced breast pathologists (E.Y.C. and S.Y.C. with 20 and 17 years of experience, respectively). After reviewing the postoperative pathology results, the final diagnoses of all lesions were categorized as DCIS or IDC (including microinvasive cancer). We also reviewed the size of invasive cancer in the surgical specimens. The patients' medical records, including pathological results, were reviewed and data were obtained on age, palpability, and procedural characteristics (guidance methods: US vS. mammography, devices: CNB vs. VAB, needle G, number of core specimens per lesion), nuclear grade, and presence of comedo necrosis from biopsy specimen ${ }^{28}$.

\section{Statistical analysis}

The potential risk factors were statistically compared between the DCIS and IDC groups using Mann-Whitney U-tests for continuous variables and chisquare or Fisher's exact tests for categorical variables. The medians and interquartile ranges (IQRs) were used for continuous variables. First, the risk of the underestimation of invasive breast cancer was analyzed using logistic regression analysis. The associations between all variables and histopathologic upgrade were evaluated using univariable logistic regression analysis. Variables showing a significant association $(P<0.05)$ in the univariable analyses were used as input variables for the multivariable logistic regression analyses. To examine the multicollinearity among these variables, we checked whether the variance inflation factor (VIF) values were 4 or higher. Multicollinearity between variables was considered when building the multivariable models. If multiple multivariable models were built, multivariable logistic regression for multiple models was performed using backward selection. The final prediction model was selected from among the candidate models, in which the lower AIC, the higher Nagelkerke $R^{2,29,30}$, the higher the AUC, and the calibration curve of the actual and predicted probabilities from the model. The AUC was computed from the original sample, from the bootstrap samples with 1000 repetitions. The bias-corrected AUC from the bootstrap samples was also calculated and a calibration plot was presented. The resulting association from the logistic regression was presented with the OR and its $95 \% \mathrm{Cl}$.

Second, three machine learning methods (decision trees, bagging, and random forests) were applied to determine whether the performance of the prediction of the estimated logistic model was reproduced. Reproducibility was checked for variable importance and AUC values. A brief description of each machine learning technique is provided in the supplementary text (online).

$P<0.05$ in the two-sided tests were considered statistically significant. All statistical analyses were performed using R (version 3.6.4; R Foundation for Statistical Computing, Vienna, Austria) or SAS (version 9.4, SAS Institute, Cary, NC, USA).

\section{Reporting Summary}

Further information on research design is available in the Nature Research Reporting Summary linked to this article.

\section{DATA AVAILABILITY}

The datasets generated during and/or analysed during the current study are not publicly available due to the institutional regulation but are available from the corresponding author on reasonable request, following ethics committee approval 
and a data transfer agreement, to guarantee the General Data Protection Regulation. Please contact the corresponding author, E.S.K. (email address: mathilda0330@gmail. com) to request access to the data.

Received: 27 May 2021; Accepted: 29 November 2021;

Published online: 14 January 2022

\section{REFERENCES}

1. Bijker, N., Donker, M., Wesseling, J., den Heeten, G. J. \& Rutgers, E. J. Is DCIS breast cancer, and how do I treat it? Curr. Treat. Options Oncol. 14, 75-87 (2013).

2. Trentin, $C$. et al. Predictors of invasive breast cancer and lymph node involvement in ductal carcinoma in situ initially diagnosed by vacuum-assisted breast biopsy: experience of 733 cases. Breast 21, 635-640 (2012).

3. Meurs, C. J. C. et al. A prediction model for underestimation of invasive breast cancer after a biopsy diagnosis of ductal carcinoma in situ: based on 2892 biopsies and 589 invasive cancers. Br. J. Cancer 119, 1155-1162 (2018).

4. Jakub, J. W. et al. A validated nomogram to predict upstaging of ductal carcinoma in situ to invasive disease. Ann. Surgical Oncol. 24, 2915-2924 (2017).

5. Kim, J. et al. Factors associated with upstaging from ductal carcinoma in situ following core needle biopsy to invasive cancer in subsequent surgical excision. Breast 21, 641-645 (2012).

6. Brennan, M. E. et al. Ductal carcinoma in situ at core-needle biopsy: meta-analysis of underestimation and predictors of invasive breast cancer. Radiology 260, 119-128 (2011).

7. National Guideline Alliance. in Early and Locally Advanced Breast Cancer: Diagnosis and Management National Institute for Health and Care Excellence: Clinical Guidelines (National Institute for Health and Care Excellence (UK, 2018).

8. Bevers, T. B. et al. Breast cancer screening and diagnosis, version 3.2018, NCCN clinical practice guidelines in oncology. J. Natl Compr. Cancer Netw. 16, 1362-1389 (2018).

9. Doyle, B. et al. Sentinel lymph node biopsy in patients with a needle core biopsy diagnosis of ductal carcinoma in situ: is it justified? J. Clin. Pathol. 62, 534-538 (2009).

10. Goyal, A. et al. Is there a role of sentinel lymph node biopsy in ductal carcinoma in situ?: analysis of 587 cases. Breast Cancer Res. Treat. 98, 311-314 (2006).

11. Li, J. et al. Predicting underestimation of ductal carcinoma in situ: a comparison between radiomics and conventional approaches. Int. J. computer Assist. Radiol. Surg. 14, 709-721 (2019).

12. Huo, L. et al. Predictors of invasion in patients with core-needle biopsy-diagnosed ductal carcinoma in situ and recommendations for a selective approach to sentinel lymph node biopsy in ductal carcinoma in situ. Cancer 107, 1760-1768 (2006).

13. Osako, T. et al. Incidence and prediction of invasive disease and nodal metastasis in preoperatively diagnosed ductal carcinoma in situ. Cancer Sci. 105, 576-582 (2014).

14. Park, H. S. et al. Risk predictors of underestimation and the need for sentinel node biopsy in patients diagnosed with ductal carcinoma in situ by preoperative needle biopsy. J. Surgical Oncol. 107, 388-392 (2013).

15. Sato, Y. et al. Preoperatively diagnosed ductal carcinoma in situ: risk prediction of invasion and effects on axillary management. Breast Cancer 23, 761-770 (2016).

16. Park, A. Y. et al. Ductal carcinoma in situ diagnosed at US-guided 14-gauge coreneedle biopsy for breast mass: preoperative predictors of invasive breast cancer. Eur. J. Radiol. 83, 654-659 (2014).

17. Bragg, A. et al. Imaging of non-calcified ductal carcinoma in situ. J. Clin. Imaging Sci. 11, 1-6 (2021).

18. Mjolsness, E. \& DeCoste, D. Machine learning for science: state of the art and future prospects. Science 293, 2051-2055 (2001).

19. Antropova, N., Huynh, B. Q. \& Giger, M. L. A deep feature fusion methodology for breast cancer diagnosis demonstrated on three imaging modality datasets. Med. Phys. 44, 5162-5171 (2017).

20. Sopik, V. et al. Impact of microinvasion on breast cancer mortality in women with ductal carcinoma in situ. Breast Cancer Res. Treat. 167, 787-795 (2018).

21. Lee, S. K., Yang, J. H., Woo, S. Y., Lee, J. E. \& Nam, S. J. Nomogram for predicting invasion in patients with a preoperative diagnosis of ductal carcinoma in situ of the breast. Br. J. Surg. 100, 1756-1763 (2013).
22. Hahn, S. Y. et al. MR features to suggest microinvasive ductal carcinoma of the breast: can it be differentiated from pure DCIS? Acta Radiol. 54, 742-748 (2013).

23. Yen, T. W. et al. Predictors of invasive breast cancer in patients with an initial diagnosis of ductal carcinoma in situ: a guide to selective use of sentinel lymph node biopsy in management of ductal carcinoma in situ. J. Am. Coll. Surg. 200, 516-526 (2005).

24. Sickles, E. A. et al. ACR BI-RADS ${ }^{\circledR}$ Atlas, Breast Imaging Reporting and Data System. 5th edn (American College of Radiology, 2013).

25. Kondo, T. et al. A model to predict upstaging to invasive carcinoma in patients preoperatively diagnosed with ductal carcinoma in situ of the breast. J. Surgical Oncol. 112, 476-480 (2015).

26. Uematsu, T. Non-mass-like lesions on breast ultrasonography: a systematic review. Breast Cancer 19, 295-301 (2012).

27. Choi, J. S. et al. Additional diagnostic value of shear-wave elastography and color Doppler US for evaluation of breast non-mass lesions detected at B-mode US. Eur. Radiol. 26, 3542-3549 (2016).

28. World Health Organisation. WHO Classification of Tumours: Breast Tumours 5th edn, Vol. 2 (International Agency for Research on Cancer, 2019)

29. Akaike, H. A new look at the statistical model identification. IEEE Trans. Autom. Control 19, 716-723 (1974).

30. Nagelkerke, N., Smits, J., le Cessie, S. \& van Houwelingen, H. Testing goodness-offit of the logistic regression model in case-control studies using sample reweighting. Stat. Med. 24, 121-130 (2005).

\section{AUTHOR CONTRIBUTIONS}

Study concept and design (E.S.K.); acquisition, analysis, or interpretation of data (all authors); drafting of manuscript (K.W.P. and E.S.K.); critical revision of the manuscript for important intellectual content (all authors).

\section{COMPETING INTERESTS}

The authors declare no competing interests.

\section{ADDITIONAL INFORMATION}

Supplementary information The online version contains supplementary material available at https://doi.org/10.1038/s41523-021-00364-z.

Correspondence and requests for materials should be addressed to Eun Sook Ko.

Reprints and permission information is available at http://www.nature.com/ reprints

Publisher's note Springer Nature remains neutral with regard to jurisdictional claims in published maps and institutional affiliations.

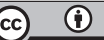

Open Access This article is licensed under a Creative Commons Attribution 4.0 International License, which permits use, sharing, adaptation, distribution and reproduction in any medium or format, as long as you give appropriate credit to the original author(s) and the source, provide a link to the Creative Commons license, and indicate if changes were made. The images or other third party material in this article are included in the article's Creative Commons license, unless indicated otherwise in a credit line to the material. If material is not included in the article's Creative Commons license and your intended use is not permitted by statutory regulation or exceeds the permitted use, you will need to obtain permission directly from the copyright holder. To view a copy of this license, visit http://creativecommons. org/licenses/by/4.0/.

(c) The Author(s) 2022 